CUBO A Mathematical Journal

Vol.18, $N^{\underline{O}} 01$, (27-45). December 2016

\title{
On generalized closed sets in generalized topological spaces
}

\author{
B. K. Tyagi ${ }^{1}$, Harsh V. S. Chauhan ${ }^{2}$ \\ 1 Department of Mathematics, \\ Atmaram Sanatan Dharma College, University of Delhi, \\ New Delhi-110021, India. \\ 2 Department of Mathematics, \\ University of Delhi, \\ New Delhi-11000\%, India \\ brijkishore.tyagi@gmail.com, harsh.chauhan111@gmail.com
}

\begin{abstract}
In this paper, we introduce several types of generalized closed sets in generalized topological spaces (GTSs). Their interrelationships are investigated and several characterizations of $\mu$ - $T_{0}, \mu-T_{1}, \mu-T_{1 / 2}, \mu$-regular, $\mu$-normal GTSs and extremally $\mu$-disconnected GTSs are obtained.
\end{abstract}

\section{RESUMEN}

En este artículo introducimos varios tipos de conjuntos cerrados generalizados en espacios topológicos generalizados (GTSs). Sus interrelaciones son investigadas y varias caracterizaciones de GTSs $\mu-T_{0}, \mu-T_{1}, \mu-T_{1 / 2}, \mu$-regulares, $\mu$-normales y extremalmente $\mu$-disconexos son obtenidas.

Keywords and Phrases: Generalized topological spaces, generalized closed sets, extremally $\mu$-disconnectedness, Separation axioms.

2010 AMS Mathematics Subject Classification: 54A05, 54D15. 


\section{Introduction}

Several types of generalized closed sets are investigated in the literature of topological spaces $[3,5,6,7,16,18,19,20,23,24,26,27,28,29,30,35,38,37,39,43,44,48,49]$. Their relationship with one another is shown by a diagram in Benchalli et al. [4] and Dontchev [17]. Using the concept of generalized closed sets, several separation axioms [17,21] are introduced which are found to be useful in the study of digital topology (digital line) [25]. Cao et al. [9] obtained several characterizations of extremally disconnectedness in terms of generalized closed sets. The purpose of this paper is to show that these diagrams can be obtained in the setting of generalized topological spaces (GTSs) introduced by Császár [11]. Let $X$ be a set and $\mathcal{P}(X)$ be the power set of $X$. A subset $\mu$ of $\mathcal{P}(X)$ is called generalized topology (GT) on $X$ if $\mu$ is closed under arbitrary unions and in that case $(X, \mu)$ is called a generalized topological space (GTS). The elements of $\mu$ are called $\mu$-open sets and their complements are called $\mu$-closed sets. The closure of $A$, denoted by $c_{\mu} A$, is the intersection of $\mu$-closed sets containing $A$. The interior of $A$, denoted by $i_{\mu} A$, is the union of $\mu$-open sets contained in $A$. In a $\operatorname{GTS}(X, \mu)$, we define $M_{\mu}=\cup\{U: U \in \mu\}$. A GTS $(X, \mu)$ is called strong if $M_{\mu}=X$.

The notions of various generalized closed sets depend on several types of stronger or weaker forms of open sets, for example, regular open set [44], semi open set [26], preopen set [31], semi preopen set [2], $\alpha$-open set [36], $\theta$-open set [50], $\delta$-open set [50], $\pi$-open set [20] etc. All these notions are extended to the setting of generalized topological spaces. The concept of $\mu-T_{1 / 2}$ GTS depends in turn on the concept of a generalized closed set. We explore the relationship of generalized closed sets with several separation axioms, $\mu-T_{0}, \mu-T_{1}, \mu-T_{1 / 2}, \mu$-regularity, and $\mu$-normality $[32,33]$.

A concept of extremally $\mu$-disconnectedness was introduced in [46]; A GTS $(X, \mu)$ is extremallay $\mu$-disconnected if $c_{\mu} \mathrm{U} \cap M_{\mu} \in \mu$ for every $\mathrm{U} \in \mu$. It may be remarked that in strong GTS, this notion concide with the notion of extremally disconnectedness in Császár [12]. Several characterizations of extremally $\mu$-disconnectedness in terms of generalized closed sets are obtained.

Section 2 contains preliminaries. In section 3, we introduce various notions of generalized closed sets and obtain several implications among them. Section 4 contains characterizations of $\mu-T_{0}, \mu-T_{1}$ and $\mu-T_{1 / 2}$ GTSs. In section 5 , we study the characterization of $\mu$-regularity and $\mu$-normality. Section 6 obtains some characterizations of extremally $\mu$-disconnected GTSs.

\section{Preliminaries}

Let $(X, \mu)$ be a GTS and $A \subseteq X . A^{c}$ denotes the complement of $A$ in $X$. The collection of all $\mu$-closed sets in $\mathrm{X}$ is denoted by $\Omega$. 
Theorem 2.1. Let $(\mathrm{X}, \mu)$ be a GTS and $\mathrm{A}, \mathrm{B} \subseteq \mathrm{X}$. Then the following statements hold.

(i) $x \in c_{\mu} A$ if and only if $x \in U \in \mu$ implies $U \cap A \neq \emptyset$.

(ii) $c_{\mu} A=c_{\mu}\left(A \cap M_{\mu}\right)$.

(iii) $c_{\mu} A=X-i_{\mu}(X-A)$.

(iv) If $\mathrm{U}, \mathrm{V} \in \mu$ and $\mathrm{U} \cap \mathrm{V}=\emptyset$ then $\mathrm{c}_{\mu} \mathrm{U} \cap \mathrm{V}=\emptyset$ and $\mathrm{U} \cap \mathrm{c}_{\mu} \mathrm{V}=\emptyset$.

(v) $M_{\mu}-c_{\mu} A=X-c_{\mu} A$.

(vi) $i_{\mu} A=i_{\mu}\left(A \cap M_{\mu}\right)$.

(vii) $i_{\mu}\left(c_{\mu} A-A\right)=\emptyset$.

(viii) $\mathrm{c}_{\mu}$ and $i_{\mu}$ are monotone: $\mathrm{A} \subseteq \mathrm{B}$ implies $\mathrm{c}_{\mu} \mathrm{A} \subseteq \mathrm{c}_{\mu} \mathrm{B}$ (respectively $i_{\mu} \mathrm{A} \subseteq i_{\mu} \mathrm{B}$ ), idempotent $c_{\mu} c_{\mu} A=c_{\mu} A$ (respectively $\left.i_{\mu} i_{\mu} A=i_{\mu} A\right), c_{\mu}$ is enhancing $\left(A \subseteq c_{\mu} A\right), i_{\mu}$ is contracting $\left(i_{\mu} A \subseteq A\right)$.

Proof. (vii). If $x \in i_{\mu}\left(c_{\mu} A-A\right)$ then there exists a $U \in \mu$ such that $x \in U \subseteq c_{\mu} A-A$. Then $x \in U \subseteq c_{\mu} A$ and $U \cap A=\emptyset$. Now $x \in U \subseteq c_{\mu} A$ implies $U \cap A \neq \emptyset$, a contradiction.

Let $(X, \mu)$ be a GTS and $Y \subseteq X$. Then the collection $\mu_{Y}=\{U \cap Y: U \in \mu\}$ is a GT on $Y$ and $\left(Y, \mu_{Y}\right)$ is called a generalized subspace of $(X, \mu)$. It may be noted that $c_{\mu_{Y}} A=c_{\mu} A \cap Y$ for any $A \subseteq Y$. Thus, a set $A \subseteq Y$ is $\mu_{Y}$-closed if and only if it is the intersection with $Y$ of a $\mu$-closed set.

Definition 2.2. A subset $\mathrm{A}$ of a $G T S(\mathrm{X}, \mu)$ is called

(i) $\mu$-regular open (or $\mathrm{ro}_{\mu}$-open) if $i_{\mu} c_{\mu} A=A$.

(ii) $\mu$-semi open (or $s_{\mu}$-open) if $A \subseteq c_{\mu} i_{\mu} A \cap M_{\mu}$.

(iii) $\mu$-preopen (or $\mathrm{p}_{\mu}$-open) if $\mathrm{A} \subseteq \mathfrak{i}_{\mu} \mathrm{c}_{\mu} \mathrm{A}$.

(iv) $\mu$ - $\alpha$-open (or $\alpha_{\mu}$-open) if $A \subseteq i_{\mu} c_{\mu} i_{\mu} A$.

(v) $\mu$-semi preopen (or $\mathrm{sp}_{\mu}$-open) if $A \subseteq \mathrm{c}_{\mu} i_{\mu} \mathrm{c}_{\mu} A \cap \mathrm{M}_{\mu}$.

(vi) $\mu$ - $\theta$-closed (or $\theta_{\mu}$-closed) [34] if $A=\gamma_{\theta} A$, where $\gamma_{\theta}(A)=\left\{x \in X: c_{\mu} G \cap M_{\mu} \cap A \neq \emptyset\right.$ for all $G \in$ $\mu, x \in \mathrm{G}\}$. The complement of a $\theta_{\mu}$-closed set is called $\mu-\theta$-open $\left(\theta_{\mu}\right.$-open).

(vii) $\mu$ - $\delta$-closed (or $\delta_{\mu}$-closed) [12] if $A=c_{\delta} A$, where $\mathrm{c}_{\delta} \mathrm{A}=\left\{\mathrm{x} \in \mathrm{X}: i_{\mu} \mathrm{c}_{\mu} \mathrm{U} \cap \mathrm{A} \neq \emptyset\right.$ for $\mathrm{U} \in \mu$ and $\left.\mathrm{x} \in \mathrm{U}\right\}$. The complement of a $\delta_{\mu}$-closed set is called $\mu$ - $\delta$-open $\left(\delta_{\mu}\right.$-open $)$. 
(viii) $\mu$ - $\pi$-open (or $\pi_{\mu}$-open) if $\mathrm{A}$ is the union of finitely many $\mu$-regular open sets.

(ix) $\mu$-regular semi open (or $\mathrm{rs}_{\mu}$-open) if there exists a $\mu$-regular open set $\mathrm{U}$ such that $\mathrm{U} \subseteq \mathrm{A} \subseteq$ $c_{\mu} \mathrm{U} \cap \mathrm{M}_{\mu}$.

The collections of all $\mu$-( ) sets in (i) to (ix) of the above definitions are denoted by $\mathrm{ro}_{\mu}, s_{\mu}$, $p_{\mu}, \alpha_{\mu}, s p_{\mu}, \theta_{\mu}, \delta_{\mu}, \pi_{\mu}, r s_{\mu}$ respectively. The complements of the sets in the above definitions are named similarly by replacing the word "open" by "closed", for example $\mu$-semi closed (or $s_{\mu^{-}}$ closed) for the complement of a $s_{\mu}$-open set and vice-versa.

It follows using Theorem 2.1, a subset $A$ of $\operatorname{GTS}(X, \mu)$ is a regular $\mu$-closed (or ro ${ }_{\mu}$-closed) if and only if $c_{\mu} i_{\mu} A=A$; $A$ set $A$ is $\mu$-semi open if and only if $c_{\mu} A=c_{\mu} i_{\mu} A$ and $A \subseteq M_{\mu}$. $A$ is $s_{\mu}$-closed if and only if $i_{\mu} c_{\mu} A \subseteq A$ and $X-M_{\mu} \subseteq A$; $A$ is $p_{\mu}$-closed if and only if $c_{\mu} i_{\mu} A \subseteq A$; $A$ is $\alpha_{\mu}$-closed if and only if $c_{\mu} i_{\mu} c_{\mu} A \subseteq A$; $A$ is $s p_{\mu}$-closed if $i_{\mu} c_{\mu} i_{\mu} A \subseteq A$ and $X-M_{\mu} \subseteq A$. For any set $A, c_{\mu} i_{\mu} c_{\mu} A$ is $\alpha_{\mu}$-closed. Also if $A \in \mathrm{rs}_{\mu}$ then $A \in s_{\mu}$ but not conversely.

Theorem 2.3. [46] For a GTS $(\mathrm{X}, \mu), \theta_{\mu}, \alpha_{\mu}, \mathrm{s}_{\mu}, \mathrm{p}_{\mu}$ and $\mathrm{sp}_{\mu}$ are GTSs and

(i) $\theta_{\mu} \subseteq \mu \subseteq \alpha_{\mu} \subseteq s_{\mu} \subseteq s p_{\mu}$,

(ii) $\alpha_{\mu} \subseteq p_{\mu} \subseteq \mathrm{sp}_{\mu}$.

Theorem 2.4. $A$ is $\alpha_{\mu}$-open if and only if $A \in s_{\mu} \cap p_{\mu}$.

Proof. If $A \subseteq i_{\mu} c_{\mu} i_{\mu} A$ then $A \subseteq c_{\mu} i_{\mu} A, A \subseteq M_{\mu}$ and $A \subseteq i_{\mu} c_{\mu} A$. So $A \in s_{\mu} \cap p_{\mu}$. Conversely, let $A \in s_{\mu} \cap p_{\mu}$. Then $A \subseteq c_{\mu} i_{\mu} A \cap M_{\mu}$. Therefore, $c_{\mu} A \subseteq c_{\mu} i_{\mu} A$. Also $A \subseteq i_{\mu} c_{\mu} A$. Therefore, $A \subseteq i_{\mu} c_{\mu} i_{\mu} A$

A subset $A$ of a $\operatorname{GTS}(X, \mu)$ is $\mu$-nowhere dense if $i_{\mu} c_{\mu} A=\emptyset$.

Lemma 2.5. Let $\chi$ be a point in a GTS $(\mathrm{X}, \mu)$. Then $\{x\}$ is $\mu$-nowhere dense or $\mathrm{p}_{\mu}$-open.

Proof. Suppose $\{x\}$ is not $\mu$-nowhere dense. Then $i_{\mu} c_{\mu}\{x\} \neq \emptyset$. Then $x \in i_{\mu} c_{\mu}\{x\}$. So $\{x\} \subseteq$ $i_{\mu} c_{\mu}\{x\}$.

Lemma 2.6. If $\{x\}$ is $\mu$-nowhere dense in a GTS $(X, \mu)$ then $\{x\} \cup\left(X-M_{\mu}\right)$ is $\alpha_{\mu}$-closed.

Proof. $c_{\mu} i_{\mu} c_{\mu}\left(\{x\} \cup\left(X-M_{\mu}\right)\right)=c_{\mu} i_{\mu} c_{\mu}\{x\}=c_{\mu} \emptyset=X-M_{\mu}$. So $c_{\mu} i_{\mu} c_{\mu}\left(\{x\} \cup\left(X-M_{\mu}\right)\right) \subseteq$ $\{x\} \cup\left(X-M_{\mu}\right)$.

Lemma 2.7. For a subset $A$ containing $X-M_{\mu}, c_{s_{\mu}} A=A \cup i_{\mu} c_{\mu} A$.

Proof. Since $c_{s_{\mu}} A$ is $s_{\mu}$-closed, $i_{\mu} c_{\mu}\left(c_{s_{\mu}} A\right) \subseteq c_{s_{\mu}} A$. On the other hand $i_{\mu} c_{\mu}\left(A \cup i_{\mu} c_{\mu} A\right) \subseteq$ $i_{\mu} c_{\mu} c_{\mu} A=i_{\mu} c_{\mu} A$. Therefore, $i_{\mu} c_{\mu}\left(A \cup i_{\mu} c_{\mu} A\right) \subseteq A \cup i_{\mu} c_{\mu} A$. Since $X-M_{\mu} \subseteq A, A \cup i_{\mu} c_{\mu} A$ is $s_{\mu}$ - closed. 
Lemma 2.8. For a subset $A, c_{\alpha_{\mu}} A=A \cup c_{\mu} i_{\mu} c_{\mu} A$.

Proof. Since $c_{\alpha_{\mu}} A$ is $\alpha_{\mu}$-closed, $c_{\mu} i_{\mu} c_{\mu} c_{\alpha_{\mu}} A \subseteq c_{\alpha_{\mu}} A$. Therefore, $A \cup c_{\mu} i_{\mu} c_{\mu} A \subseteq c_{\alpha_{\mu}} A$. On the other hand $c_{\mu} i_{\mu} c_{\mu}\left(A \cup c_{\mu} i_{\mu} c_{\mu} A\right) \subseteq c_{\mu} i_{\mu} c_{\mu} c_{\mu} A=c_{\mu} i_{\mu} c_{\mu} A \subseteq A \cup c_{\mu} i_{\mu} c_{\mu} A$. Thus, $A \cup c_{\mu} i_{\mu} c_{\mu} A$ is $\alpha_{\mu}$-closed set containing $A$.

Lemma 2.9. For a subset $A, A \cup i_{\mu} c_{\mu} i_{\mu} A \subseteq c_{s p_{\mu}} A$.

Proof. $i_{\mu} c_{\mu} i_{\mu} A \subseteq i_{\mu} c_{\mu} i_{\mu}\left(c_{s p_{\mu}}\right) A \subseteq c_{s p_{\mu}} A$, since $c_{s p_{\mu}} A$ is $s p_{\mu}$-closed.

\section{Various type of generalized closed sets}

Definition 3.1. Let $(\mathrm{X}, \mu)$ be a GTS. A subset $\mathrm{A}$ of $\mathrm{X}$ containing $\mathrm{X}-\mathrm{M}_{\mu}$ is called

(i) a $\mu$-generalized closed (or $\mathrm{g}_{\mu}$-closed) set if $\mathrm{c}_{\mu} \mathrm{A} \cap \mathrm{M}_{\mu} \subseteq \mathrm{U}$ whenever $\mathrm{A} \cap \mathrm{M}_{\mu} \subseteq \mathrm{U} \in \mu$. The complement of a $\mathrm{g}_{\mu}$-closed set is called $\mu$-generalized open (or $\mathrm{g}_{\mu}$-open). The set of all $\mathrm{g}_{\mu}$-open sets is denoted by $\mathrm{g}_{\mu}$.

(ii) a $\mu$-semi generalized closed (or $\mathrm{sg}_{\mu}$-closed) set if $\mathrm{c}_{\mathrm{s}_{\mu}} \mathrm{A} \cap \mathrm{M}_{\mu} \subseteq \mathrm{U}$ whenever $\mathrm{A} \cap \mathrm{M}_{\mu} \subseteq \mathrm{U} \in \mathrm{s}_{\mu}$.

(iii) a $\mu$-generalized semi closed (or $\mathrm{gs}_{\mu}$-closed) set if $\mathrm{c}_{\mathrm{s}_{\mu}} \mathrm{A} \cap \mathrm{M}_{\mu} \subseteq \mathrm{U}$ whenever $\mathrm{A} \cap \mathrm{M}_{\mu} \subseteq \mathrm{U} \in \mu$.

(iv) a $\mu$-generalized $\alpha$-closed (or g $\alpha_{\mu}$-closed) set if $\mathrm{c}_{\alpha_{\mu}} \mathrm{A} \cap \mathrm{M}_{\mu} \subseteq \mathrm{U}$ whenever $\mathrm{A} \cap \mathrm{M}_{\mu} \subseteq \mathrm{U} \in \alpha_{\mu}$.

(v) a $\mu \alpha$-generalized closed (or $\alpha g_{\mu}$-closed) set if $\mathrm{c}_{\alpha_{\mu}} \mathrm{A} \cap \mathrm{M}_{\mu} \subseteq \mathrm{U}$ whenever $\mathrm{A} \cap \mathrm{M}_{\mu} \subseteq \mathrm{U} \in \mu$.

(vi) a $\mu$-generalized semi preclosed (or $\mathrm{gsp}_{\mu}$-closed) set if $\mathrm{c}_{s p_{\mu}} \mathrm{A} \cap \mathrm{M}_{\mu} \subseteq \mathrm{U}$ whenever $\mathrm{A} \cap$ $\mathrm{M}_{\mu} \subseteq \mathrm{U} \in \mu$.

(vii) a $\mu$-regular generalized closed (or $\mathrm{rg}_{\mu}$-closed) set if $\mathrm{c}_{\mu} \mathrm{A} \cap \mathrm{M}_{\mu} \subseteq \mathrm{U}$ whenever $\mathrm{A} \cap \mathrm{M}_{\mu} \subseteq \mathrm{U} \in \mathrm{ro}_{\mu}$.

(viii) a $\mu$-generalized preclosed (or $\mathrm{gp}_{\mu}$-closed) set if $\mathrm{c}_{\mathrm{p}_{\mu}} \mathrm{A} \cap \mathrm{M}_{\mu} \subseteq \mathrm{U}$ whenever $\mathrm{A} \cap \mathrm{M}_{\mu} \subseteq \mathrm{U} \in \mu$.

(ix) a $\mu$-generalized preregular closed (or $\mathrm{gpr}_{\mu}$-closed) set if ${c_{p_{\mu}}} \mathrm{A} \cap \mathrm{M}_{\mu} \subseteq \mathrm{U}$ whenever $\mathrm{A} \cap$ $\mathrm{M}_{\mu} \subseteq \mathrm{U} \in \mathrm{ro}_{\mu}$.

(x) a $\mu$ - $\theta$-generalized closed (or $\theta \mathrm{g}_{\mu}$-closed) set if $\gamma_{\theta_{\mu}} \mathrm{A} \cap \mathrm{M}_{\mu} \subseteq \mathrm{U}$ whenever $\mathrm{A} \cap \mathrm{M}_{\mu} \subseteq \mathrm{U} \in \mu$.

(xi) a $\mu$ - $\delta$-generalized closed (or $\delta \mathrm{g}_{\mu}$-closed) set if $\mathrm{c}_{\delta_{\mu}} \mathrm{A} \cap \mathrm{M}_{\mu} \subseteq \mathrm{U}$ whenever $\mathrm{A} \subseteq \mathrm{U} \in \mu$.

(xii) a $\mu$-weakly generalized closed (or $w g_{\mu}$-closed) set if $\mathrm{c}_{\mu} \mathrm{i}_{\mu} A \cap M_{\mu} \subseteq \mathrm{U}$ whenever $\mathrm{A} \cap \mathrm{M}_{\mu} \subseteq \mathrm{U} \in \mu$.

(xiii) a $\mu$-strongly generalized closed (or $\mathrm{g}_{\mu}{ }^{*}$-closed) set if $\mathrm{c}_{\mu} \mathrm{A} \cap \mathrm{M}_{\mu} \subseteq \mathrm{U}$ whenever $\mathrm{A} \cap \mathrm{M}_{\mu} \subseteq \mathrm{U} \in \mathrm{g}_{\mu}$. (xiv) a $\mu$ - $\pi$-generalized closed (or $\pi \mathrm{g}_{\mu}$-closed) set if $\mathrm{c}_{\mu} \mathrm{A} \cap \mathrm{M}_{\mu} \subseteq \mathrm{U}$ whenever $\mathrm{A} \cap \mathrm{M}_{\mu} \subseteq \mathrm{U} \in \pi_{\mu}$. 
(xv) a $\mu$-weakly closed (or $w_{\mu}$-closed) set if $\mathrm{c}_{\mu} \mathrm{A} \cap \mathrm{M}_{\mu} \subseteq \mathrm{U}$ whenever $\mathrm{A} \cap \mathrm{M}_{\mu} \subseteq \mathrm{U} \in \mathrm{s}_{\mu}$.

(xvi) a $\mu$-mildly generalized closed (or $\mathrm{mg}_{\mu}$-closed) set if $\mathrm{c}_{\mu} \mathrm{i}_{\mu} \mathrm{A} \cap \mathrm{M}_{\mu} \subseteq \mathrm{U}$ whenever $\mathrm{A} \cap \mathrm{M}_{\mu} \subseteq \mathrm{U} \in \mathrm{g}_{\mu}$.

(xvii) a $\mu$-semi-weakly generalized closed (or swg $_{\mu}$-closed) set if $\mathrm{c}_{\mu} i_{\mu} A \cap M_{\mu} \subseteq U$ whenever $A \cap$ $\mathrm{M}_{\mu} \subseteq \mathrm{U} \in \mathrm{s}_{\mu}$.

(xviii) a $\mu$-regular weakly generalized closed (or $\mathrm{rwg}_{\mu}$-closed) set if $\mathrm{c}_{\mu} \mathrm{i}_{\mu} \mathrm{A} \cap \mathrm{M}_{\mu} \subseteq \mathrm{U}$ whenever $\mathrm{A} \cap \mathrm{M}_{\mu} \subseteq \mathrm{U} \in \mathrm{ro}_{\mu}$.

(xix) a $\mu$-regular generalized $w$-closed (or $\mathrm{rw}_{\mu}$-closed) set if $\mathrm{c}_{\mu} \mathrm{A} \cap \mathrm{M}_{\mu} \subseteq \mathrm{U}$ whenever $\mathrm{A} \cap \mathrm{M}_{\mu} \subseteq \mathrm{U} \in \mathrm{rs}_{\mu}$.

Lemma 3.2. (i) $A \in g_{\mu}$ then $A \subseteq M_{\mu}$.

(ii) $\mu \subseteq g_{\mu}$.

Proof. (i) Since $X-M_{\mu}$ is contained in a generalized closed set $A$, the complement of $A$ is contained in $M_{\mu}$.

(ii) Let $A \in \mu$ and $(X-A) \cap M_{\mu} \subseteq U \in \mu$. Then $c_{\mu}(X-A) \cap M_{\mu}=(X-A) \cap M_{\mu} \subseteq U$.

Theorem 3.3. A subset $\mathrm{A}$ of $G T S(\mathrm{X}, \boldsymbol{\mu})$ is $\mathrm{g}_{\mu}$-closed if and only if for any $\mu$-closed set $\mathrm{F}$ such that $\mathrm{F} \cap \mathrm{M}_{\mu} \subseteq \mathrm{c}_{\mu} \mathrm{A}-\mathrm{A}$ implies $\mathrm{F} \cap \mathrm{M}_{\mu}=\emptyset$.

Proof. Let $F$ be a $\mu$-closed set such that $F \cap M_{\mu} \subseteq c_{\mu} A-A$. Then $A \cap M_{\mu} \subseteq F^{c} \in \mu$. Since $A$ is $g_{\mu^{-}}$ closed, $c_{\mu} A \cap M_{\mu} \subseteq F^{c}$. That is, $F \cap M_{\mu} \subseteq\left(c_{\mu} A\right)^{c}$. Therefore, $F \cap M_{\mu} \subseteq\left(c_{\mu} A-A\right) \cap\left(c_{\mu} A\right)^{c}=\emptyset$. Conversely, let $A \cap M_{\mu} \subseteq U \in \mu$ and if $c_{\mu} A \cap M_{\mu}$ is not contained in $U$ then $c_{\mu} A \cap M_{\mu} \cap U^{c} \neq \emptyset$. Since $c_{\mu} A \cap U^{c}$ is $\mu$-closed and $c_{\mu} A \cap U^{\mathfrak{c}} \cap M_{\mu} \subseteq c_{\mu} A-A$, a contradiction.

Theorem 3.4. If a $\mathrm{g}_{\mu}$-closed subset $\mathrm{A}$ of a $\operatorname{GTS}(\mathrm{X}, \boldsymbol{\mu})$ be such that $\mathrm{c}_{\mu} \mathrm{A}-\left(\mathrm{A} \cap \mathrm{M}_{\mu}\right)$ is $\mu$-closed then $\mathrm{A}$ is $\mu$-closed.

Proof. Let $A$ be a $g_{\mu}$-closed set such that $c_{\mu} A-\left(A \cap M_{\mu}\right)$ is $\mu$-closed. Then $c_{\mu} A-\left(A \cap M_{\mu}\right)$ is $\mu$-closed subset of itself. Since $c_{\mu} A-\left(A \cap M_{\mu}\right)$ is $g_{\mu}$-closed subset of itself, by Theorem 3.3 $\left(c_{\mu} A-\left(A \cap M_{\mu}\right)\right) \cap M_{\mu_{Y}}$, where $Y=c_{\mu} A-\left(A \cap M_{\mu}\right)$, is empty. Since $M_{\mu_{Y}}=\left(c_{\mu} A-\left(A \cap M_{\mu}\right)\right) \cap M_{\mu}$, $A$ is $\mu$-closed.

Theorem 3.5. If $\mathrm{A}$ is a $\mathrm{g}_{\mu}$-closed set and $\mathrm{A} \subseteq \mathrm{B} \subseteq \mathrm{c}_{\mu} \mathrm{A}$ then $\mathrm{B}$ is $\mathrm{g}_{\mu}$-closed.

Proof. Let $\mathrm{B} \cap \mathrm{M}_{\mu} \subseteq \mathrm{U} \in \mu$. Since $\mathrm{A}$ is $\mathrm{g}_{\mu}$-closed and $\mathrm{A} \cap \mathrm{M}_{\mu} \subseteq \mathrm{U}, \mathrm{c}_{\mu} A \cap M_{\mu} \subseteq \mathrm{U}$. Then $\mathrm{c}_{\mu} \mathrm{B} \cap \mathrm{M}_{\mu} \subseteq \mathrm{c}_{\mu} \mathrm{A} \cap \mathrm{M}_{\mu} \subseteq \mathrm{U}$.

Theorem 3.6. In a $G T S(X, \mu), \mu=\Omega$ if and only if $(X, \mu)$ is strong and every subset of $\mathrm{X}$ is $\mathrm{g}_{\mu}$-closed. 
Proof. If $\mu=\Omega$ then obviously $(X, \mu)$ is strong. Now if $A \subseteq U \in \mu$ then $c_{\mu} A \subseteq c_{\mu} U=U$ since $\mathrm{U} \in \mu$. Conversely, let $\mathrm{U} \in \mu$. Since $\mathrm{U}$ is $\mathrm{g}_{\mu}$-closed, $\mathrm{c}_{\mu} \mathrm{U} \subseteq \mathrm{U}$. Thus, $\mathrm{U}$ is $\mu$-closed. On the other hand if $F \in \Omega$ then $F^{c} \in \mu$. Since $\mu \subseteq \Omega, F \in \mu$.

Theorem 3.7. A subset $\mathrm{A}$ of $\mathrm{M}_{\mu}$ of a $\operatorname{GTS}(\mathrm{X}, \mu)$ is $\mathrm{g}_{\mu}$-open if and only if $\mathrm{F} \cap \mathrm{M}_{\mu} \subseteq \mathfrak{i}_{\mu} \mathrm{A}$ whenever $\mathrm{F}$ is $\mu$-closed and $\mathrm{F} \cap \mathrm{M}_{\mu} \subseteq \mathrm{A}$.

Proof. Let $A$ be a $g_{\mu}$-open set and $F$ be a $\mu$-closed set such that $F \cap M_{\mu} \subseteq A$. Then $X-A \subseteq$ $X-\left(F \cap M_{\mu}\right)$. Since $(X-A) \cap M_{\mu} \subseteq\left(X-\left(F \cap M_{\mu}\right)\right) \cap M_{\mu}=X-F$ and $X-A$ is $g_{\mu}$-closed, $c_{\mu}(X-$ $A) \cap M_{\mu} \subseteq X-F$. Then $\left(X-i_{\mu} A\right) \cap M_{\mu} \subseteq X-F$. That is, $F \cap M_{\mu} \subseteq\left(X-\left(X-i_{\mu} A\right) \cap M_{\mu}\right) \cap M_{\mu}=$ $i_{\mu} A$. Conversely, let $A \subseteq M_{\mu}$ and $(X-A) \cap M_{\mu} \subseteq U \in \mu$. Then $X-U \subseteq X-\left((X-A) \cap M_{\mu}\right)$. So $(X-U) \cap M_{\mu} \subseteq A$. Then $(X-U) \cap M_{\mu} \subseteq i_{\mu} A$. So $X-i_{\mu} A \subseteq X-\left((X-U) \cap M_{\mu}\right)$. Therefore, $c_{\mu}(X-A) \cap M_{\mu} \subseteq U$. Thus, $A$ is $g_{\mu}$-open.

Theorem 3.8. A set $A$ in $G T S(X, \mu)$ is $g_{\mu}$-open if and only if $i_{\mu} A \cup\left(A^{c} \cap M_{\mu}\right) \subseteq U \in \mu$ implies $\mathrm{U}=\mathrm{M}_{\mu}$.

Proof. Let $A$ be a $g_{\mu}$-open set and $i_{\mu} A \cup\left(A^{c} \cap M_{\mu}\right) \subseteq U \in \mu$. Then $U^{c} \subseteq\left(i_{\mu} A\right)^{c} \cap\left(A \cup M_{\mu}^{c}\right)=$ $c_{\mu}(X-A) \cap\left(A \cup M_{\mu}^{c}\right)$. Therefore, $U^{c} \cap M_{\mu} \subseteq\left(c_{\mu}(X-A) \cap M_{\mu}\right) \cap A=c_{\mu}(X-A)-(X-A)$. Then by Theorem $3.3 \mathrm{U}^{\mathfrak{c}} \cap M_{\mu}=\emptyset$, That is, $U=M_{\mu}$. Conversely, let $F$ be a $\mu$-closed set such that $F \cap M_{\mu} \subseteq A$. Then $i_{\mu} A \cup\left(A^{c} \cap M_{\mu}\right) \subseteq i_{\mu} A \cup F^{c} \in \mu$. By the assumption, $i_{\mu} A \cup F^{c}=M_{\mu}$, that is, $F \cap M_{\mu} \subseteq i_{\mu} A$. Now apply Theorem 3.7.

Theorem 3.9. A subset $\mathrm{A}$ of a $\operatorname{GTS}(\mathrm{X}, \mu)$ is $\mathrm{g}_{\mu}$-closed if and only if $\mathrm{c}_{\mu} \mathrm{A}-\mathrm{A}$ is $\mathrm{g}_{\mu}$-open.

Proof. Suppose that $A$ is $g_{\mu}$-closed and $F \cap M_{\mu} \subseteq c_{\mu} A-A$, where $F$ is a $\mu$-closed set. By Theorem 3.3 $F \cap M_{\mu}=\emptyset$. So $F \cap M_{\mu} \subseteq i_{\mu}\left(c_{\mu} A-A\right)$. Therefore, $c_{\mu} A-A$ is $g_{\mu^{-}}$open by Theorem 3.7. Conversely, assume that $X-M_{\mu} \subseteq A$ and $A \cap M_{\mu} \subseteq U \in \mu$. Now $c_{\mu} A \cap U^{c} \cap M_{\mu} \subseteq$ $c_{\mu} A \cap\left(M_{\mu}-A\right)=c_{\mu} A-A$. By Theorem $3.7 c_{\mu} A \cap U^{c} \cap M_{\mu} \subseteq i_{\mu}\left(c_{\mu} A-A\right)=\emptyset$. Thus, $c_{\mu} A \cap M_{\mu} \subseteq U$ and $A$ is $g_{\mu}$-closed.

The following diagram extends to the setting of GTSs the corresponding diagram of Benchalli and Wali [4] and Dontchev [17]. 


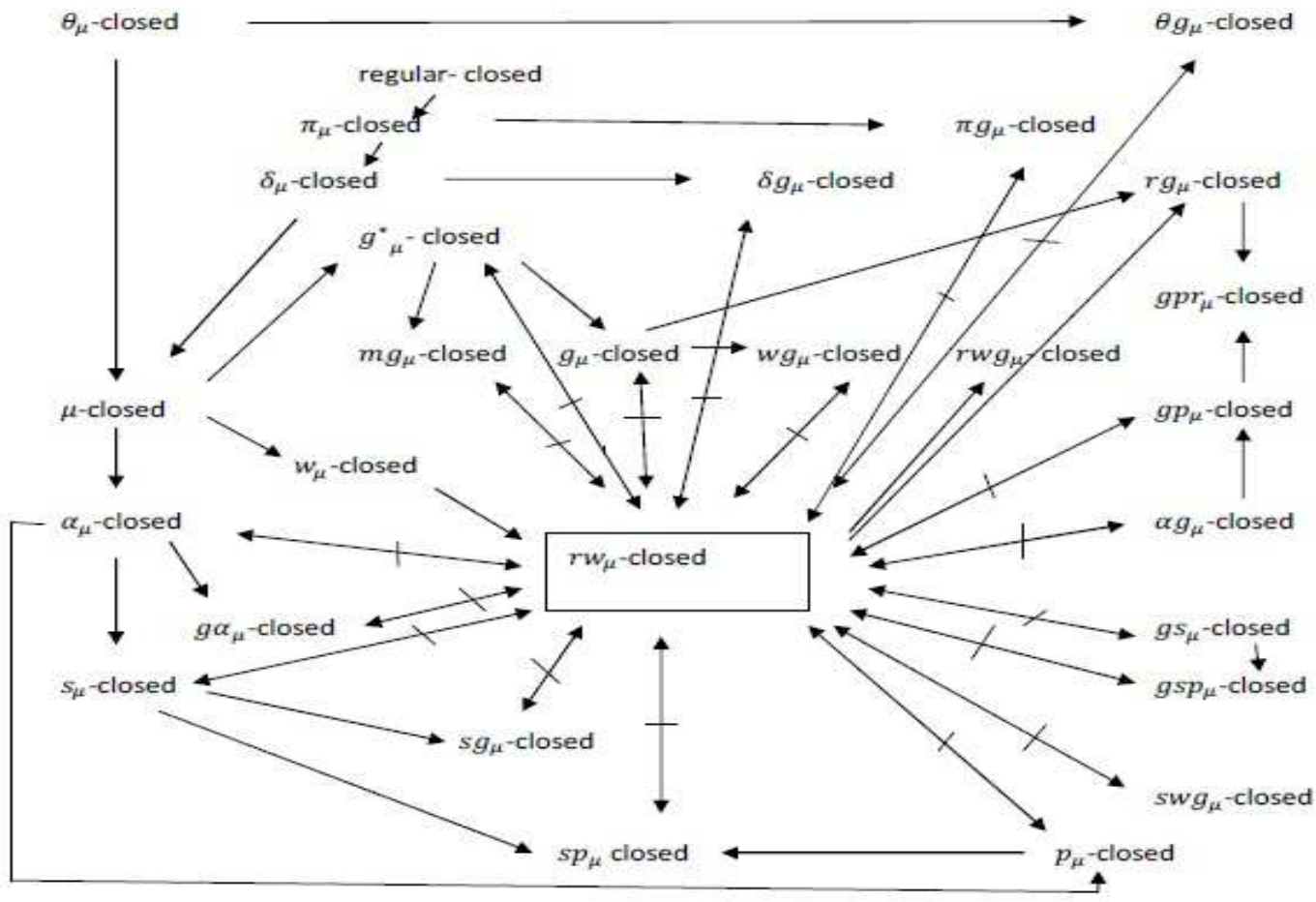

$\mathrm{A} \longrightarrow \mathrm{B}$ means A implies B but not conversely

$\mathrm{A} \longleftrightarrow 1 \longrightarrow$ B means $\mathrm{A}$ and $\mathrm{B}$ are independent to each other

For examples showing independence $A \leftrightarrow B$ in the above diagram see [4].

Theorem 3.10. Let $(\mathrm{X}, \mu)$ be a GTS and $\mathrm{A} \subseteq \mathrm{X}$. Then the following statements hold.

(i) $\mu$-closed $\Rightarrow \alpha_{\mu}$-closed $\Rightarrow s_{\mu}$-closed $\Rightarrow$ sp $_{\mu}$-closed.

(ii) $\alpha_{\mu}$-closed $\Rightarrow \mathrm{p}_{\mu}$-closed $\Rightarrow \mathrm{sp}_{\mu}$-closed.

(iii) $\mu$-closed $\Rightarrow \mathrm{g}_{\mu}$-closed $\Rightarrow \mathrm{rg}_{\mu}$ - closed.

(iv) $\mathrm{g}_{\mu}$-closed $\Rightarrow \alpha \mathrm{g}_{\mu}$-closed $\Rightarrow \mathrm{gs}_{\mu}$-closed $\Rightarrow \mathrm{gsp}_{\mu}$-closed.

(v) $\alpha_{\mu}$-closed $\Rightarrow g \alpha_{\mu}$-closed $\Rightarrow \alpha g_{\mu}$-closed.

(vi) $\mathrm{s}_{\mu}$-closed $\Rightarrow \mathrm{sg}_{\mu}$-closed $\Rightarrow \mathrm{gsp}_{\mu}$-closed.

(vii) $\mathrm{sg}_{\mu}$-closed $\Rightarrow \mathrm{gs}_{\mu}$-closed.

(viii) $\mathrm{sp}_{\mu}$-closed $\Rightarrow \mathrm{gsp}_{\mu}$-closed. 
(ix) $\mathrm{p}_{\mu}$-closed $\Rightarrow \mathrm{gsp} \mathrm{p}_{\mu}$-closed.

(x) $\alpha g_{\mu}$-closed $\Rightarrow \mathrm{gsp}_{\mu}$-closed.

(xi) $\mathrm{g} \alpha_{\mu}$-closed $\Rightarrow \mathrm{gs}{ }_{\mu}$-closed.

Proof. (i) Let $A$ be $\mu$-closed set. Then $c_{\mu} A=A$. Therefore, $i_{\mu} c_{\mu} A=i_{\mu} A \subseteq A$. Thus, $c_{\mu} i_{\mu} c_{\mu} A \subseteq c_{\mu} A=A$.

Now let $A$ be a $\alpha_{\mu}$-closed set. Then $i_{\mu} c_{\mu} A \subseteq c_{\mu} i_{\mu} c_{\mu} A \subseteq A$.

Now let $A$ be a $s_{\mu}$-closed set. Then $i_{\mu} c_{\mu} A \subseteq A$ and $X-M_{\mu} \subseteq A$. Therefore, $i_{\mu} c_{\mu} i_{\mu} A \subseteq$ $i_{\mu} c_{\mu} A \subset A$. This proves (i).

The proofs of other parts also follow easily.

Theorem 3.11. (i) Every $\mathrm{sg}_{\mu}$-closed sets is $\mathrm{sp}_{\mu}$-closed.

(ii) Every $g \alpha_{\mu}$-closed set is $p_{\mu}$-closed.

Proof. (i) Let $A$ be a $s g_{\mu}$-closed set and $x \in c_{s p_{\mu}} A \cap M_{\mu}$. Then $\{x\}$ is $p_{\mu}$-open or $\mu$-nowhere dense. If $\{x\}$ is $p_{\mu}$-open then by Theorem 2.3, \{x\} is $\operatorname{sp}_{\mu}$-open. Since $x \in s_{\mu} A \cap M_{\mu}$, $\{x\} \cap A \neq \emptyset$. Therefore, $x \in A$. If $\{x\}$ is $\mu$-nowhere dense then $\{x\} \cup\left(X-M_{\mu}\right)$ is $\alpha_{\mu}$-closed and hence $s_{\mu}$-closed. Therefore, the complement $B=M_{\mu}-\{x\}$ is $s_{\mu}$-open. Assume that $x \notin A$, then $A \cap M_{\mu} \subseteq B$. Since $A$ is $s_{\mu_{\mu}}$-closed, and $c_{s p_{\mu}} A \subseteq c_{s_{\mu}} A . c_{s p_{\mu}} A \cap M_{\mu} \subseteq B$. Hence $x \notin c_{s p_{\mu}} A \cap M_{\mu}$. By contradiction $x \in A$. Thus, $A$ is $s p_{\mu}$-closed.

(ii) Let $A$ be a $g \alpha_{\mu}$-closed set. Let $x \in c_{p_{\mu}} A \cap M_{\mu}$. If $\{x\}$ is $p_{\mu}$-open, then $\{x\} \cap A \neq \emptyset$. So that $x \in A$. If $\{x\}$ is $\mu$-nowhere dense and does not meet $A$ then $\{x\} \cup\left(X-M_{\mu}\right)$ is $\alpha_{\mu}$-closed. Then $B=M_{\mu}-\{x\}$ is $\alpha_{\mu}$-open and $A \cap M_{\mu} \subseteq B$. Since $A$ is $g \alpha_{\mu}$-closed, $c_{\alpha \mu} A \cap M_{\mu} \subseteq B$. Therefore, $x \notin c_{\alpha \mu} A \cap M_{\mu}$, a contradiction. Thus, $x \in A$ and $A$ is $p_{\mu}$-closed.

The following theorem also covers some immediate implications.

Theorem 3.12. For a set in a GTS $(\mathrm{X}, \mu)$, the following statements hold.

(i) $\pi_{\mu}$-closed $\Rightarrow \delta_{\mu}$-closed.

(ii) $\theta_{\mu}$-closed $\Rightarrow \theta g_{\mu}$-closed.

(iii) $\pi_{\mu}$-closed $\Rightarrow \pi g_{\mu}$-closed.

(iv) $\delta_{\mu}$-closed $\Rightarrow \delta g_{\mu}$-closed.

(v) $\mu$-closed $\Rightarrow \mathrm{g}_{\mu}{ }^{*}$-closed.

(vi) $\mu$-closed $\Rightarrow w_{\mu}$-closed. 
(vii) $\mathrm{g}_{\mu}{ }^{*}$-closed $\Rightarrow \mathrm{mg}_{\mu}$-closed.

(viii) $\mathrm{g}_{\mu}{ }^{*}$-closed $\Rightarrow \mathrm{g}_{\mu}$-closed.

(ix) $\mathrm{g}_{\mu}-$ closed $\Rightarrow w \mathrm{~g}_{\mu}$-closed.

(x) $\mathrm{rg}_{\mu}$-closed $\Rightarrow \mathrm{gpr}_{\mu}$-closed.

(xi) $\mathrm{gp}_{\mu}$-closed $\Rightarrow \mathrm{gpr}_{\mu}$-closed.

(xii) $\alpha g_{\mu}$-closed $\Rightarrow g p_{\mu}$-closed.

(xiii) $w_{\mu}$-closed $\Rightarrow$ rw $_{\mu}$-closed.

(xiv) $\mathrm{rw}_{\mu}$-closed $\Rightarrow \mathrm{rg}_{\mu}$-closed.

$(\mathrm{xv}) \mathrm{rw}_{\mu}$-closed $\Rightarrow \mathrm{rwg}_{\mu}$-closed.

Proof. (i) Let $A$ be a $\pi_{\mu}$-closed set. Then there are $\mu$-regular closed sets $R_{i}, R_{2}, \ldots . . R_{n}$ such that $A=\bigcap_{i=1}^{n} R_{i}$. Let $x \in X-A=\cup_{i=1}^{n} R_{i}{ }^{c}$. Then $x \in R_{i}{ }^{c}$ for some $i$ and $i_{\mu} c_{\mu} R_{i}{ }^{c} \cap A=$ $R_{i}{ }^{c} \cap A=\emptyset$. So $x \notin c_{\delta \mu} A$.

The proofs of other parts are also easy and left to the reader.

\section{$4 \mu-T_{0}, \mu-T_{1}$ and $\mu-T_{1 / 2}$ generalized topological spaces}

Definition 4.1. A GTS $(\mathrm{X}, \mu)$ is said to be

(i) $\mu-T_{0}$ if $x, y \in M_{\mu}, x \neq y$ implies the existence of a $\mu$-open set containing precisely one of $x$ and $y$.

(ii) [32] $\mu$ - $\mathrm{T}_{1}$ if $\mathrm{x}, \mathrm{y} \in \mathrm{M}_{\mu}, \mathrm{x} \neq \mathrm{y}$ implies the existence of $\mu$-open sets $\mathrm{U}_{1}$ and $\mathrm{U}_{2}$ such that $\mathrm{x} \in \mathrm{U}_{1}$ and $\mathrm{y} \notin \mathrm{U}_{1}$ and $\mathrm{y} \in \mathrm{U}_{2}$ and $\mathrm{x} \notin \mathrm{U}_{2}$.

(iii) $\mu-\mathrm{T}_{1 / 2}$ if every $\mathrm{g}_{\mu}$-closed set is $\mu$-closed.

Easy examples of GT-spaces which are not strong and having the properties of the above separation axioms may be provided. For example, let $R$ be the set of real numbers and $x, y, x \neq y$ be any two real numbers. Then $\mu=\{\emptyset,\{x\},\{x, y\}\}$ is a GT which is not strong and has the property of $\mu-T_{0}$ but not $\mu-T_{1}$.

It is obvious that $\mu-T_{1}$ implies $\mu-T_{0}$. Also $(X, \mu)$ is $\mu$ - $T_{0}$ if and only if for each $x, y \in M_{\mu}$, $c_{\mu}(\{x\})=c_{\mu}(\{y\})$ implies $x=y$.

Theorem 4.2. If a $\operatorname{GTS}(\mathrm{X}, \mu)$ is $\mu-\mathrm{T}_{1 / 2}$ then it is $\mu-\mathrm{T}_{0}$. 
Proof. Suppose that $(X, \mu)$ is not a $\mu$-To space. Then there exist distinct points $x$ and $y$ in $M_{\mu}$ such that $c_{\mu}(\{x\})=c_{\mu}(\{y\})$. Let $A=c_{\mu}(\{x\}) \cap\{x\}^{c}$. We show that $A$ is $g_{\mu}$-closed but not $\mu$-closed. $X-M_{\mu} \subseteq A$. Let $A \cap M_{\mu} \subseteq V \in \mu$. Since $A \subseteq c_{\mu}(\{x\}), c_{\mu} A \cap M_{\mu} \subseteq c_{\mu}(\{x\}) \cap M_{\mu}$. Thus, we show that $c_{\mu}(\{x\}) \cap M_{\mu} \subseteq V$. Since $c_{\mu}(\{x\}) \cap\{x\}^{c} \cap M_{\mu} \subseteq V$, it is enough to show that $x \in V$. If $x$ is not in $\mathrm{V}$ then $\mathrm{y} \in \mathrm{V}$ and $\mathrm{y} \in \mathrm{c}_{\mu}(\{y\})=\mathrm{c}_{\mu}(\{x\}) \subseteq \mathrm{V}^{\mathfrak{c}}$ as $\mathrm{V}^{\mathfrak{c}}$ is a $\mu$-closed set containing the set $\{x\}$. Thus, $y \in V \cap V^{c}$, a contradiction. Now if $x \in U \in \mu$ then $U \cap A \supseteq\{y\} \neq \emptyset$, and hence $x \in c_{\mu} A$. But $x$ is not in $A$ and thus, $A$ is not a $\mu$-closed set.

Theorem 4.3. If a GTS $(X, \mu)$ is $\mu$ - $T_{1}$ then for each $\chi \in X, A=\{x\} \cup\left(X-M_{\mu}\right)$ is $\mu$-closed.

Proof. Let $y \in c_{\mu} A \cap M_{\mu}$ and $y \neq x$. Then $y \in c_{\mu}\left(A \cap M_{\mu}\right) \cap M_{\mu}=c_{\mu}(\{x\}) \cap M_{\mu}$. Then $y \in c_{\mu}(\{x\})$. So $y \in U \in \mu$ implies $x \in U$ which is against our hypothesis. So $c_{\mu} A \cap M_{\mu}=\{x\}$, that is, $c_{\mu} A=A$.

Theorem 4.4. If a $\operatorname{GTS}(\mathrm{X}, \mu)$ is $\mu-\mathrm{T}_{1}$ then it is $\mu-\mathrm{T}_{1 / 2}$.

Proof. Let $A$ be a subset of $X$ which is not $\mu$-closed. If $X-M_{\mu}$ is not contained in $A$, then $A$ is not $g_{\mu}$-closed. So let $X-M_{\mu} \subseteq A$. Since $A$ is not $\mu$-closed, $c_{\mu} A-A$ is non empty. Let $x \in c_{\mu} A-A$. By Theorem $4.3\{x\} \cup\left(X-M_{\mu}\right)$ is $\mu$-closed. As $\left(\{x\} \cup\left(X-M_{\mu}\right)\right) \cap M_{\mu}=\{x\} \subseteq c_{\mu} A-A$, by Theorem $3.3 \mathrm{~A}$ is not $\mathrm{g}_{\mu}$-closed.

Definition 4.5. A GTS $(\mathrm{X}, \mu)$ is said to be $\mu$-symmetric if for each $\mathrm{x}, \mathrm{y} \in \mathrm{M}_{\mu}, \mathrm{x} \in \mathrm{c}_{\mu}(\{\mathrm{y}\})$ implies $y \in c_{\mu}(\{x\})$.

Theorem 4.6. A GTS $(\mathrm{X}, \mu)$ is $\mu$-symmetric if and only if $\{x\} \cup\left(\mathrm{X}-\mathrm{M}_{\mu}\right)$ is $\mathrm{g}_{\mu}$-closed for each $x \in X$.

Proof. Let $A=\{x\} \cup\left(X-M_{\mu}\right)$ and $A \cap M_{\mu} \subseteq U \in \mu$. If $A \cap M_{\mu}=\emptyset$ then $c_{\mu} A=c_{\mu}\left(A \cap M_{\mu}\right)=$ $c_{\mu} \emptyset=X-M_{\mu}$. So $c_{\mu} A \cap M_{\mu} \subseteq U$. Otherwise $c_{\mu} A \cap M_{\mu}=c_{\mu}\left(A \cap M_{\mu}\right) \cap M_{\mu}=c_{\mu}(\{x\}) \cap M_{\mu}$. If $c_{\mu}(\{x\}) \cap M_{\mu} \nsubseteq U$ then assume that $y \in c_{\mu}(\{x\}) \cap U^{c} \cap M_{\mu}$. Since $(X, \mu)$ is $\mu$-symmetric, $x \in c_{\mu}(\{y\})$. Since $x \in U, y \in U$, then $y \in U \cap U^{c}$, a contradiction. Conversely, let for each $x \in X,\{x\} \cup\left(X-M_{\mu}\right)$ is $g_{\mu}$-closed. Let $x, y \in M_{\mu}, x \in c_{\mu}(\{y\})$ and $y \notin c_{\mu}(\{x\})$. Then $y \in\left(c_{\mu}(\{x\})\right)^{c}$. Let $A=\{y\} \cup\left(X-M_{\mu}\right)$. Then $A$ is $g_{\mu}$-closed and $A \cap M_{\mu}=\{y\} \subseteq\left(c_{\mu}(\{x\})\right)^{c}$. So $c_{\mu} A \cap M_{\mu}=$ $\left(c_{\mu}(\{y\})\right) \cap M_{\mu} \subseteq\left(c_{\mu}(\{x\})\right)^{c}$. Then $x \in\left(c_{\mu}(\{y\})\right) \cap M_{\mu} \subseteq\left(c_{\mu}(\{x\})\right)^{c}$, a contradiction.

Corollary 4.7. If A GTS $(\mathrm{X}, \mu)$ is $\mu-\mathrm{T}_{1}$ then it is $\mu$-symmetric.

Proof. The proof follows from Theorem 4.3, Theorem 4.6 and Lemma 3.2.

Theorem 4.8. A GTS $(\mathrm{X}, \mu)$ is $\mu$-symmetric and $\mu-\mathrm{T}_{0}$ if and if only $(\mathrm{X}, \mu)$ is $\mu-\mathrm{T}_{1}$. 
Proof. If $(X, \mu)$ is $\mu$ - $T_{1}$ then by Corollary $4.7(X, \mu)$ is $\mu$-symmetric and obviously $\mu$ - $T_{0}$. Conversely, let $(X, \mu)$ be $\mu$-symmetric and $\mu-T_{0}$. Let $x, y \in M_{\mu}$ and $x \neq y$. Then by $\mu$ - $T_{0}$ property there exists a $U \in \mu$ such that $x \in U \subseteq(\{y\})^{c}$. Then $x$ is not in $c_{\mu}(\{y\})$. Since $(X, \mu)$ is $\mu$-symmetric, $y$ is not in $c_{\mu}(\{x\})$. Then there exists $V=\left(c_{\mu}(\{x\})\right)^{c}$ such that $y \in V$ and $x \notin V$.

Theorem 4.9. If $(\mathrm{X}, \mu)$ is $\mu$-symmetric then $(\mathrm{X}, \mu)$ is $\mu$ - $\mathrm{T}_{0}$ if and only if $(\mathrm{X}, \mu)$ is $\mu-\mathrm{T}_{1 / 2}$ if and only if $(\mathrm{X}, \mu)$ is $\mu-\mathrm{T}_{1}$.

Proof. The proof follows from Theorems 4.8, 4.4 and 4.2.

Theorem 4.10. A GTS $(\mathrm{X}, \mu)$ is $\mu-\mathrm{T}_{1 / 2}$ if and only if for each $\mathrm{x} \in \mathrm{X}$, either $\{\mathrm{x}\}$ is $\mu$-open or $\{x\} \cup\left(\mathrm{X}-\mathrm{M}_{\mu}\right)$ is $\mu$-closed.

Proof. Suppose $X$ is $\mu-T_{1 / 2}$ and for some $x \in X,\{x\} \cup\left(X-M_{\mu}\right)$ is not $\mu$-closed. Then $M_{\mu}$ is the only $\mu$-open set containing $M_{\mu}-\{x\}$. Therefore, $\left(M_{\mu}-\{x\}\right) \cup\left(X-M_{\mu}\right)$ is $g_{\mu}$-closed. So it is $\mu$-closed. Thus, $\{x\}$ is $\mu$-open.

Conversely, let $A$ be a $g_{\mu}$-closed set with $x \in c_{\mu} A \cap M_{\mu}$ and $x \notin A$. If $\{x\}$ is $\mu$-open then $\emptyset \neq\{x\} \cap A$. Thus, $x \in A$. Otherwise $\{x\} \cup\left(X-M_{\mu}\right)$ is $\mu$-closed. Then $\left(\{x\} \cup\left(X-M_{\mu}\right)\right) \cap M_{\mu}=$ $\{x\} \subseteq c_{\mu} A-A$. Then by Theorem $3.3\{x\}=\emptyset$, a contradiction. Thus, $x \in A$ and so $A$ is $\mu$-closed.

Theorem 4.11. For a GTS $(\mathrm{X}, \mu)$, the following statements are equivalent.

(i) $\mathrm{X}$ is $\mu-\mathrm{T}_{1 / 2}$.

(ii) Every $\alpha g_{\mu}$-closed set is $\alpha_{\mu}$-closed.

Proof. $(i) \Rightarrow$ (ii). Let $A$ be a $\alpha g_{\mu}$-closed set and $x \in c_{\alpha_{\mu}} A \cap M_{\mu}$. If $\{x\}$ is $\mu$-open then $\{x\} \in \alpha_{\mu}$ so that $\{x\} \cap A \neq \emptyset$. Thus, $x \in A$. Otherwise $\{x\} \cup\left(X-M_{\mu}\right)$ is $\mu$-closed. Let $x \notin A$. Then $M_{\mu}-\{x\}$ is $\mu$-open and $A \cap M_{\mu} \subseteq M_{\mu}-\{x\}$. Since $A$ is $\alpha g_{\mu}$-closed, $c_{\alpha_{\mu}} A \cap M_{\mu} \subseteq M_{\mu}-\{x\}$. Therefore, $x \notin c_{\alpha_{\mu}} A \cap M_{\mu}$, a contradiction. Thus, $x \in A$ and $A$ is $\alpha_{\mu}$-closed.

(ii) $\Rightarrow(i)$. If some set $\{x\} \cup\left(X-M_{\mu}\right)$ is not $\mu$-closed then $x \in M_{\mu}$ and $M_{\mu}-\{x\}$ is not $\mu$-open. Then $\left(M_{\mu}-\{x\}\right) \cup\left(X-M_{\mu}\right)$ is trivially $\alpha g_{\mu}$-closed. By (ii), $\left(M_{\mu}-\{x\}\right) \cup\left(X-M_{\mu}\right)$ is $\alpha_{\mu}$-closed. So $\{x\}$ is $\alpha_{\mu}$-open. Since a non-empty $\alpha_{\mu}$-open set contains a non-empty $\mu$-open set, $\{x\}$ is $\mu$-open. This shows that $(X, \mu)$ is $\mu-T_{1 / 2}$.

\section{$5 \quad \mu$-regular and $\mu$-normal generalized topological spaces}

Definition 5.1. [33] A GTS $(\mathrm{x}, \mu)$ is said to be $\mu$-regular if for each $\mu$-closed set $\mathrm{F}$ of $\mathrm{X}$ not containing $\mathrm{x} \in \mathrm{X}$ there exist disjoint $\mu$-open subsets $\mathrm{U}$ and $\mathrm{V}$ of $\mathrm{X}$ such that $\mathrm{x} \in \mathrm{U}$ and $\mathrm{F} \cap \mathrm{M}_{\mu} \subseteq \mathrm{V}$.

Theorems 5.2, 5.4, and 5.5 generalize the corresponding results in Roy [40]. 
Theorem 5.2. For a GTS $(\mathrm{X}, \mu)$, the following statements are equivalent.

(i) $\mathrm{X}$ is $\mu$-regular.

(ii) $\mathrm{x} \in \mathrm{U} \in \mu$ implies that there exists $\mathrm{V} \in \mu$ such that $\mathrm{x} \in \mathrm{V} \subseteq \mathrm{c}_{\mu} \mathrm{V} \cap \mathrm{M}_{\mu} \subseteq \mathrm{U}$.

(iii) For each $\mu$-closed set $\mathrm{F}, \mathrm{F}=\cap\left\{\mathrm{c}_{\mu} \mathrm{V}: \mathrm{F} \cap \mathrm{M}_{\mu} \subseteq \mathrm{V} \in \mu\right\}$.

(iv) For each subset $\mathrm{A}$ of $\mathrm{X}$ and each $\mathrm{U} \in \mu$ with $\mathrm{A} \cap \mathrm{U} \neq \emptyset$ there exists $a \mathrm{~V} \in \mu$ such that $\mathrm{A} \cap \mathrm{V} \neq \emptyset$ and $\mathrm{c}_{\mu} \mathrm{V} \cap \mathrm{M}_{\mu} \subseteq \mathrm{U}$.

( $v$ ) For each non-empty set $\mathrm{A} \subseteq \mathrm{X}$ and each $\mu$-closed set $\mathrm{F}$ with $\mathrm{A} \cap \mathrm{F}=\emptyset$ there exist $\mathrm{U}, \mathrm{V} \in \mu$ such that $\mathrm{A} \cap \mathrm{V} \neq \emptyset, \mathrm{F} \cap \mathrm{M}_{\mu} \subseteq \mathrm{U}$ and $\mathrm{U} \cap \mathrm{V}=\emptyset$.

(vi) For each $\mu$-closed set $\mathrm{F}$ and $\mathrm{x} \notin \mathrm{F}$ there exist $\mathrm{U} \in \mu$ and a $\mathrm{g}_{\mu}$-open set $\mathrm{V}$ such that $\mathrm{x} \in \mathrm{U}$, $\mathrm{F} \cap \mathrm{M}_{\mu} \subseteq \mathrm{V}$ and $\mathrm{U} \cap \mathrm{V}=\emptyset$.

(vii) For each non-empty $\mathrm{A} \subseteq \mathrm{X}$ and each $\mu$-closed set $\mathrm{F}$ with $\mathrm{A} \cap \mathrm{F}=\emptyset$ there exist $a \mathrm{U} \in \mu$ and $a$ $\mathrm{g}_{\mu}$-open set $\mathrm{V}$ such that $\mathrm{A} \cap \mathrm{U} \neq \emptyset, \mathrm{F} \cap \mathrm{M}_{\mu} \subseteq \mathrm{V}$ and $\mathrm{U} \cap \mathrm{V}=\emptyset$.

(viii) For each $\mu$-closed set $\mathrm{F}$ of $\mathrm{X}, \mathrm{F}=\cap\left\{\mathbf{c}_{\mu} \mathrm{V}: \mathrm{F} \cap \mathrm{M}_{\mu} \subseteq \mathrm{V}\right.$ and $\mathrm{V}$ is $\mathrm{g}_{\mu}$-open $\}$.

Proof. (i) $\Leftrightarrow$ (ii) [32].

(ii) $\Rightarrow$ (iii). Suppose $x \notin F$. Then by (ii) there exists a $V \in \mu$ such that $x \in V \subseteq c_{\mu} V \cap M_{\mu} \subseteq X-F$. Then $F \cap M_{\mu} \subseteq\left(X-\left(c_{\mu} V \cap M_{\mu}\right)\right) \cap M_{\mu}=X-c_{\mu} V=W \in \mu$. Since $c_{\mu} W \cap V=\emptyset$, (iii) follows. (iii) $\Rightarrow$ (iv). $x \in A \cap U$ implies that $x \notin X-U$. By (iii) there exists a $W \in \mu$ such that $(X-U) \cap$ $M_{\mu} \subseteq W$ and $x \notin c_{\mu} W$. Let $V=X-c_{\mu} W$ then $x \in V \cap A$ and $V \subseteq X-W$. Thus, $c_{\mu} V \subseteq X-W$. Therefore, $c_{\mu} V \cap M_{\mu} \subseteq(X-W) \cap M_{\mu} \subseteq\left(X-\left((X-U) \cap M_{\mu}\right)\right) \cap M_{\mu}=U$.

(iv) $\Rightarrow(\mathrm{v})$. $A \cap(X-F) \neq \emptyset$. By (iv) there exists a $\mu$-open set $V$ such that $A \cap V \neq \emptyset$ and $c_{\mu} V \cap M_{\mu} \subseteq X-F$. Let $W=X-c_{\mu} V$. Then $F \cap M_{\mu} \subseteq \subseteq\left(X-\left(c_{\mu} \vee \cap M_{\mu}\right)\right) \cap M_{\mu}=X-c_{\mu} V=W$ and $\mathrm{W} \cap \mathrm{V}=\emptyset$.

(v) $\Rightarrow$ (i). Let $F$ be a $\mu$-closed set not containing $x$. By (v) there exist disjoint $\mu$-open sets $U$ and $\mathrm{V}$ such that $\mathrm{x} \in \mathrm{U}$ and $\mathrm{F} \cap \mathrm{M}_{\mu} \subseteq \mathrm{V}$.

(i) $\Rightarrow$ (vi). Follows from Lemma 3.2 .

(vi) $\Rightarrow$ (vii). Note that $A \subseteq M_{\mu}$. Since $A$ is non-empty and $A \cap F=\emptyset$ there exists a point $x \in A$ such that $x \notin \mathrm{F}$. By (vi) there exist a $\mathrm{U} \in \mu$ and a $\mathrm{g}_{\mu}$-open set $\mathrm{V}$ such that $\mathrm{x} \in \mathrm{U}, \mathrm{F} \cap \mathrm{M}_{\mu} \subseteq \mathrm{V}$ and $\mathrm{U} \cap \mathrm{V}=\emptyset$. Then $\mathrm{U} \cap \mathrm{A} \neq \emptyset$.

(vii) $\Rightarrow$ (i). Let $x \notin F$, where $F$ is a $\mu$-closed set. Then $\{x\} \cap F=\emptyset$. By (vii) there exist a $U \in \mu$ and a $g_{\mu}$-open set $\mathrm{V}$ such that $x \in \mathrm{U}, \mathrm{F} \cap \mathrm{M}_{\mu} \subseteq \mathrm{V}$ and $\mathrm{U} \cap \mathrm{V}=\emptyset$. Now $\mathrm{F} \cap \mathrm{M}_{\mu} \subseteq i_{\mu} \mathrm{V}$ by Theorem 3.7. (iii) $\Rightarrow$ (viii). We have $\mathrm{F} \subseteq \cap\left\{\mathrm{c}_{\mu} \mathrm{V}: \mathrm{F} \cap \mathrm{M}_{\mu} \subseteq \mathrm{V}\right.$ and $\mathrm{V}$ is $\mathrm{g}_{\mu}$-open $\} \subseteq \cap\left\{\mathrm{c}_{\mu} \mathrm{V}: \mathrm{F} \cap \mathrm{M}_{\mu} \subseteq \mathrm{V} \in \mu\right\}=\mathrm{F}$. (viii) $\Rightarrow$ (i). Let $F$ be a $\mu$-closed set such that $x \notin F$. Then by (viii) there exists $g_{\mu}$-open set $W$ such that $F \cap M_{\mu} \subseteq W$ and $x \notin c_{\mu} W$. Since $F$ is $\mu$-closed, $W$ is $g_{\mu}$-open and $F \cap M_{\mu} \subseteq W$, by Theorem 3.7, $\mathrm{F} \cap \mathrm{M}_{\mu} \subseteq \mathfrak{i}_{\mu} W$. 
Definition 5.3. [32] A GTS $(\mathrm{X}, \mu)$ is $\mu$-normal if for any pair of $\mu$-closed sets $\mathrm{A}$ and $\mathrm{B}$ such that $\mathrm{A} \cap \mathrm{B} \cap \mathrm{M}_{\mu}=\emptyset$ there exist disjoint $\mu$-open sets $\mathrm{U}$ and $\mathrm{V}$ such that $\mathrm{A} \cap \mathrm{M}_{\mu} \subseteq \mathrm{U}$ and $\mathrm{B} \cap \mathrm{M}_{\mu} \subseteq \mathrm{V}$.

Theorem 5.4. For a $\operatorname{GTS}(\mathrm{X}, \mu)$, the following statements are equivalent.

(i) $\mathrm{X}$ is $\mu$-normal.

(ii) For any $\mu$-closed set $\mathrm{A}$ and $\mu$-open set $\mathrm{U}$ such that $\mathrm{A} \cap \mathrm{M}_{\mu} \subseteq \mathrm{U}$ there is a $\mu$-open set $\mathrm{V}$ such that $\mathrm{A} \cap \mathrm{M}_{\mu} \subseteq \mathrm{V} \subseteq \mathrm{c}_{\mu} \mathrm{V} \cap \mathrm{M}_{\mu} \subseteq \mathrm{U}$.

Proof. Let $A$ be a $\mu$-closed set such that $A \cap M_{\mu} \subseteq U \in \mu$. Then $B=X-U$ is $\mu$-closed and $A \cap B \cap M_{\mu}$ is empty. Then by (i) there exist disjoint $\mu$-open sets $V$ and $W$ such that $A \cap M_{\mu} \subseteq V$ and $\mathrm{B} \cap M_{\mu} \subseteq W$. Then $A \cap M_{\mu} \subseteq \mathrm{V} \subseteq \mathrm{c}_{\mu} \mathrm{V} \cap \mathrm{M}_{\mu} \subseteq(\mathrm{X}-\mathrm{W}) \cap \mathrm{M}_{\mu} \subseteq\left(\mathrm{X}-\left(\mathrm{B} \cap \mathrm{M}_{\mu}\right)\right) \cap \mathrm{M}_{\mu}=\mathrm{U}$. Conversely, assume that $A$ and $B$ be $\mu$-closed sets such that $A \cap B \cap M_{\mu}=\emptyset$. Then $U=X-B$ is $\mu$ open and $A \cap M_{\mu} \subseteq \mathrm{U}$. By (ii) there exists a $\mu$-open set $\mathrm{V}$ such that $A \cap M_{\mu} \subseteq \mathrm{V} \subseteq \mathrm{C}_{\mu} \mathrm{V} \cap \mathrm{M}_{\mu} \subseteq \mathrm{U}$. Let $W=X-c_{\mu} V$. Since $c_{\mu} V \cap B \cap M_{\mu}=\emptyset, B \cap M_{\mu} \subseteq X-c_{\mu} V=W$.

Theorem 5.5. In a GTS $(\mathrm{X}, \mu)$, the following statements are equivalent.

(i) $\mathrm{X}$ is $\mu$-normal.

(ii) For any pair of $\mu$-closed sets $A$ and $B$ such that $A \cap B \cap M_{\mu}=\emptyset$ then there exist disjoint $\mathrm{g}_{\mu}$-open sets $\mathrm{U}$ and $\mathrm{V}$ such that $\mathrm{A} \cap \mathrm{M}_{\mu} \subseteq \mathrm{U}$ and $\mathrm{B} \cap \mathrm{M}_{\mu} \subseteq \mathrm{V}$.

(iii) For every $\mu$-closed set $\mathrm{A}$ and $\mu$-open set $\mathrm{U}$ such that $\mathrm{A} \cap \mathrm{M}_{\mu} \subseteq \mathrm{U}$ there exists a $\mathrm{g}_{\mu}$-open set $\mathrm{V}$ such that $\mathrm{A} \cap \mathrm{M}_{\mu} \subseteq \mathrm{V} \subseteq \mathrm{c}_{\mu} \mathrm{V} \cap \mathrm{M}_{\mu} \subseteq \mathrm{U}$.

(iv) For every $\mu$-closed set $\mathrm{A}$ and every $\mathrm{g}_{\mu}$-open set $\mathrm{U}$ containing $\mathrm{A} \cap \mathrm{M}_{\mu}$ there exists a $\mu$-open set $\mathrm{V}$ such that $\mathrm{A} \cap \mathrm{M}_{\mu} \subseteq \mathrm{V} \subseteq \mathrm{c}_{\mu} \mathrm{V} \cap \mathrm{M}_{\mu} \subseteq \mathrm{U}$.

( $v)$ For every $\mathrm{g}_{\mu}$-closed set $\mathrm{A}$ and every $\mu$-open set $\mathrm{U}$ containing $\mathrm{A} \cap \mathrm{M}_{\mu}$ there exists a $\mu$-open set $\mathrm{V}$ such that $\mathrm{c}_{\mu} \mathrm{A} \cap \mathrm{M}_{\mu} \subseteq \mathrm{V} \subseteq \mathrm{c}_{\mu} \mathrm{V} \cap \mathrm{M}_{\mu} \subseteq \mathrm{U}$.

Proof. (i) $\Rightarrow$ (ii). Follows from Lemma 3.2.

(ii) $\Rightarrow$ (iii). Assume that $B=X-U$. By (ii) there exist disjoint $g_{\mu}$-open sets $V$ and $W$ such that $A \cap M_{\mu} \subseteq V$ and $B \cap M_{\mu} \subseteq W$. Since $B \cap M_{\mu} \subseteq W,(X-U) \cap M_{\mu} \subseteq W$. Therefore, $(X-W) \cap M_{\mu} \subseteq\left(X-(X-U) \cap M_{\mu}\right) \cap M_{\mu}=U$. Since $X-W$ is $g_{\mu}$-closed, $c_{\mu}(X-W) \cap M_{\mu} \subseteq U$. Since $c_{\mu} V \cap M_{\mu} \subseteq c_{\mu}(X-W) \cap M_{\mu}$, the implication is established.

(iii) $\Rightarrow$ (iv). By Theorem $3.7 A \cap M_{\mu} \subseteq i_{\mu} U$. Then by (iii) there exists a $g_{\mu}$-open set $V$ such that $A \cap M_{\mu} \subseteq \mathrm{V} \subseteq \mathrm{c}_{\mu} \mathrm{V} \cap \mathrm{M}_{\mu} \subseteq i_{\mu} \mathrm{U}$. By Theorem $3.7 A \cap \mathrm{M}_{\mu} \subseteq i_{\mu} \mathrm{V} \subseteq \mathrm{c}_{\mu}\left(i_{\mu} \mathrm{V}\right) \cap \mathrm{M}_{\mu} \subseteq$ $\mathrm{c}_{\mu} \mathrm{V} \cap \mathrm{M}_{\mu} \subseteq \mathrm{U}$.

(iv) $\Rightarrow(\mathrm{v})$. Let $A$ be a $g_{\mu}$-closed set and $A \cap M_{\mu} \subseteq U \in \mu$. Then $c_{\mu} A \cap M_{\mu} \subseteq U$. By (iv) there exists a $\mu$-open set $\mathrm{V}$ such that $\mathrm{c}_{\mu} A \cap \mathrm{M}_{\mu} \subseteq \mathrm{V} \subseteq \mathrm{c}_{\mu} \mathrm{V} \cap \mathrm{M}_{\mu} \subseteq \mathrm{U}$.

(v) $\Rightarrow$ (i). Let $A$ and $B$ be $\mu$-closed sets such that $A \cap B \cap M_{\mu}=\emptyset$. Then $A \cap M_{\mu} \subseteq X-B \in \mu$. By 
(v) there exists a $\mu$-open set $\mathrm{V}$ such that $\mathrm{c}_{\mu} \mathrm{A} \cap \mathrm{M}_{\mu} \subseteq \mathrm{V} \subseteq \mathrm{c}_{\mu} \mathrm{V} \cap \mathrm{M}_{\mu} \subseteq \mathrm{X}-\mathrm{B}$. Thus, $\mathrm{A} \cap \mathrm{M}_{\mu} \subseteq \mathrm{V}$ and $\mathrm{B} \subseteq \mathrm{X}-\left(\mathrm{c}_{\mu} \mathrm{V} \cap \mathrm{M}_{\mu}\right)$. Therefore, $\mathrm{B} \cap \mathrm{M}_{\mu} \subseteq\left(\mathrm{X}-\left(\mathrm{c}_{\mu} \mathrm{V} \cap \mathrm{M}_{\mu}\right)\right) \cap \mathrm{M}_{\mu}=\mathrm{X}-\mathrm{c}_{\mu} \mathrm{V}=\mathrm{W} \in \mu$.

\section{$6 \quad$ Extremally $\mu$-disconnectedness}

Theorem 6.1. For a $G T S(\mathrm{X}, \mu)$, the following statements are equivalent.

(i) $(\mathrm{X}, \mu)$ is extremally $\mu$-disconnected.

(ii) Every $\mathrm{sp}_{\mu}$-closed set is $\mathrm{p}_{\mu}$-closed.

(iii) Every $\mathrm{sg}_{\mu}$-closed set is $\mathrm{p}_{\mu}$-closed.

(iv) Every $\mathrm{s}_{\mu}$-closed set is $\mathrm{p}_{\mu}$-closed.

( $v)$ Every $\mathrm{s}_{\mu}$-closed set is $\alpha_{\mu}$-closed.

(vi) Every $\mathrm{s}_{\mu}$-closed set is $\mathrm{g} \alpha_{\mu}$-closed.

Proof. $(i) \Rightarrow$ (ii). Let $A$ be a $\operatorname{sp}_{\mu}$-closed set. Then by Lemma $2.9 i_{\mu} c_{\mu} i_{\mu} A \subseteq A$. Since $X$ is extremally $\mu$-disconnected, $c_{\mu} i_{\mu} A \cap M_{\mu}=i_{\mu}\left(c_{\mu} i_{\mu} A \cap M_{\mu}\right) \subseteq i_{\mu} c_{\mu} i_{\mu} A$. Therefore, $c_{\mu} i_{\mu} A \cap M_{\mu} \subseteq$ A. Since $X-M_{\mu} \subseteq A, c_{\mu} i_{\mu} A \subseteq A$.

(ii) $\Rightarrow$ (iii). is Theorem 3.11(i).

$(\mathfrak{i i i}) \Rightarrow(\mathfrak{i v})$. Since a $\mathrm{s}_{\mu}$-closed set is $\mathrm{sg}_{\mu^{-}}$closed, the result follows.

$(i v) \Rightarrow(v)$. Follows from Theorem 2.4 .

$(v) \Rightarrow(v i)$. follows from Theorem $3 \cdot 10(v)$.

$(v i) \Rightarrow(i)$. Let $U$ be a $\mu$-open set. We need to show that $i_{\mu}\left(c_{\mu} U \cap M_{\mu}\right)=c_{\mu} U \cap M_{\mu}$. Now $i_{\mu}\left(c_{\mu} U \cap M_{\mu}\right)=i_{\mu} c_{\mu} U$. Since $i_{\mu} c_{\mu} U \subseteq c_{\mu} U \cap M_{\mu}$, we prove the inclusion $c_{\mu} U \cap M_{\mu} \subseteq i_{\mu} c_{\mu} U$. Let $A=i_{\mu} c_{\mu} U \cup X-M_{\mu}$. Now $i_{\mu} c_{\mu} A=i_{\mu} c_{\mu} i_{\mu} c_{\mu} U=i_{\mu} c_{\mu} U \subseteq A$. So $A$ is $s_{\mu}$-closed. By our assumption $A$ is $g \alpha_{\mu}$-closed. Since $i_{\mu} c_{\mu} i_{\mu}\left(i_{\mu} c_{\mu} U\right)=i_{\mu} c_{\mu} U, i_{\mu} c_{\mu} U$ is $\alpha_{\mu}$-open. Since $A \cap M_{\mu}=$ $i_{\mu} c_{\mu} U \in \alpha_{\mu}$ and $A$ is $g \alpha_{\mu}$-closed, $c_{\alpha_{\mu}} A \cap M_{\mu} \subseteq i_{\mu} c_{\mu} U \subseteq A$. Thus, $c_{\alpha_{\mu}} A \subseteq A$. On the other hand $c_{\alpha_{\mu}} A=A \cup c_{\mu} i_{\mu} c_{\mu} A$ implies $c_{\mu} i_{\mu} c_{\mu} A \subseteq A$. Therefore, $c_{\mu} i_{\mu} c_{\mu} A \cap M_{\mu} \subseteq A \cap M_{\mu}=i_{\mu} c_{\mu} A$, which implies that $A$ is $\mu$-closed. Now $U \subseteq i_{\mu} c_{\mu} U$. Then $c_{\mu} U \subseteq c_{\mu} i_{\mu} c_{\mu} U=c_{\mu} A=A$. Therefore, $c_{\mu} \mathrm{U} \cap \mathrm{M}_{\mu} \subseteq i_{\mu} c_{\mu} \mathrm{U}$.

Future scope: This paper may be useful in the study of digital topology since generalized closed sets and $\mathrm{T}_{1 / 2}$ separation axiom have already proved their utility in that area. 


\section{Acknowledgements}

The second author acknowledges the fellowship grant of University Grant Commission, India. The authors are very grateful to anonymous referee for his observations which improved the paper.

\section{References}

[1] M.E. Abd El-Monsef, S.N. El-Deep and R.A. Mahmoud, $\beta$-open sets and $\beta$-continuous mappings, Bull. Fac. Sci. Assiut Univ. 12 (1983), 77-90.

[2] D. Andrijevic, Semi-preopen sets Mat. Vesnik 38 (1986), 24-32.

[3] S.P. Arya and T.M. Nour, Characterizations of s-normal spaces, Indian J. Pure Appl. Math, 21 (1990), 717-719.

[4] S.S. Benchalli and R.S. Wali, On RW- closed sets in topological spaces Bull. Malays. Math. sci. soc. 30(2) (2007), 99-110.

[5] P. Bhattacharyya and B.K. Lahiri, Semi-generalized closed sets in topology, Indian J. Math. 29 (1987), 376-382.

[6] N. Biswas, On characterization of semi-continuous functions, Atti Accad. Naz. Lincei Rend, Cl. Sci. Fis. Mat. Natur 48 (8)(1970), 399-402.

[7] D.E. Cameron, Properties of S-closed spaces, Proc. Amer Math. soc. 72 (1978), 581-586.

[8] J. Cao, M. Ganster, Submaximal, extremal disconnectedness and generalized closed sets, Houston Journal of Mathematics 24(4) (1998), 681-688.

[9] J. Cao, M. Ganster, I. Reilly, On generalized closed sets, Topology and its application 123 (2002), 17-47.

[10] Á. Császár, Generalized open sets, Acta Math. Hungar. 75 (1997), 65-87.

[11] Á. Császár, Generalized topology, generalized continuity, Acta Math. Hungar. 96 (2002), 351-357.

[12] Á. Császár, $\delta$ and $\theta$ modification topologies, Annales Univ. Sci. Budapest 47 (2004), 91-96.

[13] Á. Császár, Separation axioms for generalized topologies, Acta Math. Hungar. 104 (2004), 63-69.

[14] Á. Császár, Normal generalized topologies, Acta Math. Hungar. 115(4) (2007), 309-313.

[15] Á. Császár, Extremally disconnected generalized topologies, Acta Math. Hungar. 120 (2008), 275-279. 
[16] J. Dontchev, On generalizing semi-preopen sets, Mem. Fac Sci. Kochi. Univ. Ser. A. Math. 16 (1995), 35-48.

[17] J. Dontchev, On some separation axioms associated with $\alpha$ - topology, Mem. Fac Sci. Kochi. Univ. Ser. A. Math. 18 (1997), 31-35.

[18] J. Dontchev and M. Ganster, On $\delta$ - generalized set T $3 / 4$ Spaces, Mem. Fac Sci. Kochi. Univ. Ser. A. Math. 17 (1996), 15-31.

[19] J. Dontchev and H. Maki, On $\theta$-generalized closed sets, Topology Atlass, www.Unipissing.ca/topology/p/a/b/a/08.htm.

[20] J. Dontchev and T. Noiri, Quasi-normal spaces and $\pi$ g-closed sets, Acta Math. Hungar. 89(3) (2000), 211-219.

[21] W. Dunham, $\mathrm{T}_{1 / 2}$ spaces, Kyungpook Math. J. 17(2) (1997), 161-169.

[22] E. Ekici, On $\gamma$ - normal space, Bull. Math. soc. Math. Roumanie. Tome 50(98)(3) (2007), 259-272.

[23] Y. Gnanambal, On generalized preregular closed sets in topological spaces, Indian J. Pure Appl. Math. 28 (1997), 351-360.

[24] Y. Gnanambal and K. Balachandran, On gpr-continuous functions in topological spaces, Indian J. Pure Appl. Math. 30(6) (1999), 581-593.

[25] E.D. Khalimsky, Applications of connected ordered topological spaces in topology, Conference of Math. Department of Povolsia, 1970.

[26] N. Levine, semi open sets and semi-continuity in topological spaces, Amer. Math. Monthly 70 (1963), 36-41.

[27] N. Levine, Generalized closed sets in topology, Rend. Circ. Mat. Palermo 19 (1970), 89-96.

[28] H. Maki, R. Devi and K. Balachandran, Generalized $\alpha$-closed set in topology, Bull. Fukuoka Univ. Ed. part-III 42 (1993), 13-21.

[29] H. Maki, R. Devi and K. Balachandran, Associated topologies of generalized $\alpha$ - closed sets and $\alpha$-generalized closed sets, Mem. Sci. Kochi. Univ. Ser. A. Math. 15 (1994), 51-63.

[30] H. Maki, J. Umehara and T. Noiri, Every topological space is pri- $1_{1 / 2}$, Mem. Fac Sci. Kochi. Univ. Ser. A. Math. 17 (1996), 33-42.

[31] A.S. Mashhour, M.E. Abd. El-Monsef and S. N. El-deep, On pre continuous mappings and weak pre-continuous mappings Proc Math. Phys. Soc. Egypt 53 (1982), 47-53.

[32] W.K. Min, Remarks on separation axioms on generalized topological space, Chungcheong mathematical society 23(2) (2010), 293-298. 
[33] W.K. Min, $\left(\delta, \delta^{\prime}\right)$-continuity on generalized topological spaces, Acta Math. Hungar. 129(4) (2010), 350-356.

[34] W.K. Min, Remark on $\theta$ - open sets in generalized topological spaces, Applied math. letter's 24 (2011), 165-168.

[35] N. Nagaveni, Studies on generalizations of homemorphisms in topological spaces, Ph.D. Thesis, Bharathiar University, Coimbatore, 1999.

[36] O. Njastad, On some classes of nearly open sets, Pacific J. Math. 15 (1965), 961-970.

[37] N. Palaniappan and K.C. Rao, Regular generalized closed sets, Kyungpook Math J. 33 (1993), 211-219.

[38] J.K. Park and J.H. Park, Mildly generalized closed sets, almost normal and mildly normal spaces, Chaos, Solitions and Fractals 20 (2004), 1103-1111.

[39] A. Pushpalatha, Studies on generalizations of mappings in topological spaces, Ph.D. Thesis, Bharathiar University, Coimbatore, 2000.

[40] B. Roy, On a type of generalized open sets, Applied general topology 12(2) (2011), 163-173.

[41] R.D. Sarma, On extremally disconnected generalized topologies Acta Math. Hungar. 134(4) (2012), 583-588.

[42] M. S. Sarsak, Weak separation axioms in generalized topological spaces, Acta Math. Hungar. 131(1-2) (2011), 110-121.

[43] P. Sundaram and M. Sheik John, On w-closed sets in topology, Acta Ciencia Indica 4 (2000), 389-392.

[44] M. Stone, Application of the theory of Boolean rings to generalized topology Trans. Amer. Math. Soc. 41 (1937), 374-481.

[45] B.K. Tyagi, H.V.S. Chauhan, A remark on semi open sets in generalized topological spaces, communicated.

[46] B.K. Tyagi, H.V.S. Chauhan, A remark on exremally $\mu$-disconnected generalized topological spaces, Mathematics for applications, 5 (2016), 83-90.

[47] B.K. Tyagi, H.V.S. Chauhan, R. Choudhary On $\gamma_{\theta}$-operator and $\theta$-connected sets in generalized topological space, Journal of Advanced Studies in Topology 6(4) (2015), 135-143.

[48] J. Tong, Weak almost continuous mapping and weak nearly compact spaces, Boll. Un. Mat. Ital. 6(1982), 385-391.

[49] M.K.R.S. Veera Kumar, Between closed sets and g-closed sets, Mem. Fac Sci. Kochi. Univ.(Math) 21 (2000), 1-19. 
[50] N.V. Velicko, H-closed topological space, Trans. Amer. Math. Soc. 78 (1968), 103-118.

[51] GE Xun, GE Ying, $\mu$-separations in generalized topological spaces, Appl. Math. J. Chiness Univ. 25(2) (2010), 243-252. 